\title{
Inclusive charm production in two-photon collisions at LEP
}

\section{L3 Collaboration}

M. Acciarri z , P. Achard ', O. Adriani ', M. Aguilar-Benitez ${ }^{y}$, J. Alcaraz ${ }^{y}$, G. Alemanni ", J. Allaby p, A. Aloisio ${ }^{\text {ab }}$, M.G. Alviggi ab , G. Ambrosi ', H. Anderhub au, V.P. Andreev f,aj, T. Angelescu l, F. Anselmo i, A. Arefiev aa, T. Azemoon ${ }^{\text {c }}$, T. Aziz ${ }^{\text {j, P. Bagnaia ai }}{ }^{\text {, L. Baksay }}{ }^{\text {ap }}$, A. Balandras ${ }^{\text {d }}$, R.C. Ball ${ }^{\text {c }}$, S. Banerjee ${ }^{j}$, Sw. Banerjee ${ }^{j}$, K. Banicz ${ }^{\text {ar }}$, A. Barczyk ${ }^{\text {au,as }}$, R. Barillère ${ }^{p}$, L. Barone ai, P. Bartalini ", M. Basile ${ }^{\text {i }, ~ R . ~ B a t t i s t o n ~}{ }^{\text {af }}$, A. Bay ", F. Becattini ${ }^{\circ}$, U. Becker ${ }^{\mathrm{n}}$, F. Behner ${ }^{\text {au }}$, J. Berdugo ${ }^{\mathrm{y}}$, P. Berges ${ }^{\mathrm{n}}$, B. Bertucci ${ }^{\text {af }}$, B.L. Betev ${ }^{\text {au }}$, S. Bhattacharya ${ }^{j}$, M. Biasini af ${ }^{\text {, A. Biland }}{ }^{\text {au }}$, J.J. Blaising ${ }^{\mathrm{d}}$, S.C. Blyth ${ }^{\mathrm{ag}}$, G.J. Bobbink ${ }^{\text {b }}$, R. Bock ${ }^{\text {a }}$, A. Böhm ${ }^{\text {a }}$, L. Boldizsar ${ }^{\mathrm{m}}$, B. Borgia ${ }^{\text {pai }}$, D. Bourilkov ${ }^{\text {au }}$, M. Bourquin ', S. Braccini ', J.G. Branson al, V. Brigljevic au, F. Brochu ${ }^{\text {d, }}$ A. Buffini ${ }^{\circ}$, A. Buijs ${ }^{\text {aq }}$, J.D. Burger ${ }^{n}$, W.J. Burger ${ }^{\text {af }}$, J. Busenitz ${ }^{\text {ap }}$, A. Button ${ }^{c}$, X.D. Cai " , M. Campanelli ${ }^{\text {au }}$, M. Capell n, G. Cara Romeo i, G. Carlino ${ }^{\text {ab }}$, A.M. Cartacci ${ }^{\circ}$, J. Casaus ${ }^{y}$, G. Castellini ${ }^{\circ}$, F. Cavallari ai, N. Cavallo ${ }^{\text {ab }}$, C. Cecchi ' , M. Cerrada y , F. Cesaroni v, M. Chamizo ${ }^{y}$, Y.H. Chang ${ }^{\text {aw }}$, U.K. Chaturvedi ${ }^{q}$, M. Chemarin ${ }^{\mathrm{x}}$, A. Chen ${ }^{\text {aw }}$, G. Chen ${ }^{\mathrm{g}}$, G.M. Chen ${ }^{\mathrm{g}}$, H.F. Chen ${ }^{\text {s, H.S. Chen }}{ }^{\mathrm{g}}$, X. Chereau ${ }^{\mathrm{d}}$, G. Chiefari ${ }^{\mathrm{ab}}$, L. Cifarelli ${ }^{\mathrm{a}}$, F. Cindolo ${ }^{\mathrm{i}}$,

C. Civinini ${ }^{o}$, I. Clare ${ }^{n}$, R. Clare ${ }^{n}$, G. Coignet ${ }^{\text {d }}$, A.P. Colijn ${ }^{\text {b }}$, N. Colino ${ }^{y}$, S. Costantini ${ }^{\mathrm{h}}$, F. Cotorobai ${ }^{1}$, B. de la Cruz ${ }^{\mathrm{y}}$, A. Csilling ${ }^{\mathrm{m}}$, T.S. Dai ${ }^{\mathrm{n}}$, J.A. van Dalen ${ }^{\text {ad }}$, R. D’Alessandro ${ }^{\circ}$, R. de Asmundis ${ }^{\text {ab }}$, P. Deglon ${ }^{\mathrm{r}}$, A. Degré ${ }^{\mathrm{d}}$, K. Deiters ${ }^{\text {as }}$, D. della Volpe ${ }^{\text {ab }}$, P. Denes ${ }^{\text {ah }}$, F. DeNotaristefani a ${ }^{\text {a }}$, A. De Salvo ${ }^{\text {au }}$, M. Diemoz ai, D. van Dierendonck ${ }^{\text {b }}$, F. Di Lodovico ${ }^{\text {au }}$, C. Dionisi ${ }^{\text {pai }}$, M. Dittmar ${ }^{\text {au }}$, A. Dominguez ${ }^{\text {al }}$, A. Doria ${ }^{\text {ab }}$, M.T. Dova ${ }^{\text {q,1 }}$, D. Duchesneau ${ }^{\text {d, }}$ D. Dufournand ${ }^{\text {d }}$, P. Duinker b , I. Duran ${ }^{\text {am }}$, H. El Mamouni ${ }^{x}$, A. Engler ${ }^{\mathrm{ag}}$, F.J. Eppling ", F.C. Erné b, P. Extermann ' , M. Fabre as, R. Faccini ai, M.A. Falagan ${ }^{y}$, S. Falciano ${ }^{\text {ai }}$, A. Favara ${ }^{\circ}$, J. Fay ${ }^{x}$, O. Fedin ${ }^{\text {aj }}$, M. Felcini ${ }^{\text {au }}$, T. Ferguson ${ }^{\text {ag }}$, F. Ferroni ${ }^{\text {ai }}$, H. Fesefeldt ${ }^{\text {a }}$, E. Fiandrini ${ }^{\text {af }}$, J.H. Field ${ }^{\mathrm{r}}$, F. Filthaut ${ }^{\text {p }}$, P.H. Fisher ${ }^{n}$, I. Fisk ${ }^{\text {al }}$, G. Forconi ${ }^{n}$, L. Fredj ${ }^{\mathrm{r}}$, K. Freudenreich ${ }^{\text {au }}$, C. Furetta $^{\text {z, Yu. Galaktionov }}{ }^{\text {aa, }}$, S.N. Ganguli ${ }^{\text {j, }}$ P. Garcia-Abia ${ }^{\text {e }}$, M. Gataullin ${ }^{\text {ae }}$, 
S.S. Gau ${ }^{\text {k }}$, S. Gentile ai , N. Gheordanescu ${ }^{1}$, S. Giagu ${ }^{\text {ai }}$, S. Goldfarb ", Z.F. Gong s, M.W. Gruenewald ${ }^{\text {h }}$, R. van Gulik ${ }^{\text {b }}$, V.K. Gupta ${ }^{\text {ah }}$, A. Gurtu ${ }^{\mathrm{j}}$, L.J. Gutay ${ }^{\text {ar }}$, D. Haas ${ }^{\text {e }}$, B. Hartmann ${ }^{\text {a }}$, A. Hasan ${ }^{\text {ac }}$, D. Hatzifotiadou ${ }^{\text {i }}$, T. Hebbeker ${ }^{\text {h }}$, A. Hervé ${ }^{p}$, P. Hidas ${ }^{m}$, J. Hirschfelder ${ }^{a g}, H$. Hofer ${ }^{\text {au }}$, G. Holzner ${ }^{\text {au }}$, H. Hoorani ${ }^{\text {ag }}$, S.R. Hou ${ }^{\text {aw }}$, I. Iashvili at, B.N. Jin ${ }^{\mathrm{g}}$, L.W. Jones ${ }^{\mathrm{c}}$, P. de Jong ${ }^{\mathrm{p}}$, I. Josa-Mutuberría $^{\text {y }}$, R.A. Khan ${ }^{\mathrm{q}}$, D. Kamrad ${ }^{\text {at }}$, J.S. Kapustinsky ${ }^{\text {w }}$, M. Kaur ${ }^{\text {q,2 }}$, M.N. Kienzle-Focacci ${ }^{\text {r }}$, D. Kim ${ }^{\text {ai }}$, D.H. Kim ${ }^{\text {ao }}$, J.K. Kim ${ }^{\text {ao }}$, S.C. Kim ${ }^{\text {ao }}$, W.W. Kinnison ${ }^{\text {w }}$, J. Kirkby ${ }^{\mathrm{p}}$, D. Kiss ${ }^{\mathrm{m}}$, W. Kittel ${ }^{\text {ad }}$, A. Klimentov ${ }^{\text {n,aa }}$, A.C. König ${ }^{\text {ad }}$, A. Kopp ${ }^{\text {at }}$, I. Korolko aa , V. Koutsenko ${ }^{\text {naa }}$, R.W. Kraemer ${ }^{\text {ag }}$, W. Krenz ${ }^{\text {a }}$, A. Kunin ${ }^{\text {n,aa }}$, P. Lacentre ${ }^{\text {at, } 1,3}$, P. Ladron de Guevara ${ }^{y}$, I. Laktineh ${ }^{\text {x }}$, G. Landi ${ }^{\circ}$, C. Lapoint ${ }^{n}$, K. Lassila-Perini ${ }^{\text {au }}$, P. Laurikainen ${ }^{\mathrm{t}}$, A. Lavorato ${ }^{\mathrm{ak}}$, M. Lebeau ${ }^{\mathrm{p}}$, A. Lebedev ${ }^{\mathrm{n}}$, P. Lebrun ${ }^{\mathrm{x}}$, P. Lecomte ${ }^{\mathrm{au}}$, P. Lecoq ${ }^{\mathrm{p}}$, P. Le Coultre ${ }^{\mathrm{au}}$, H.J. Lee ${ }^{\text {h }}$, J.M. Le Goff ${ }^{\mathrm{p}}$, R. Leiste ${ }^{\text {at }}$, E. Leonardi ${ }^{\text {ai }}$, P. Levtchenko ${ }^{\text {aj }}$, C. Li ${ }^{\text {s }}$, C.H. Lin ${ }^{\text {aw }}$, W.T. Lin ${ }^{\text {aw }}$, F.L. Linde ${ }^{\text {b,p }}$, L. Lista ${ }^{\text {ab }}$, Z.A. Liu ${ }^{\mathrm{g}}$, W. Lohmann ${ }^{\text {at }}$, E. Longo ${ }^{\text {ai }}$, Y.S. Lu ${ }^{g}$, K. Lübelsmeyer a , C. Luci ${ }^{\text {pai }}$, D. Luckey ${ }^{\text {n }}$, L. Luminari ai, W. Lustermann ${ }^{\text {au }}$, W.G. Ma ${ }^{\text {s }}$, M. Maity ${ }^{j}$, G. Majumder ${ }^{j}$, L. Malgeri ${ }^{\text {p }}$, A. Malinin aa, C. Maña ${ }^{\text {y }}$, D. Mangeol ad, P. Marchesini ${ }^{\text {au }}$, G. Marian ${ }^{\text {ap }, 4}$, J.P. Martin ${ }^{\mathrm{x}}$, F. Marzano ${ }^{\text {ai }}$, G.G.G. Massaro ${ }^{\mathrm{b}}$, K. Mazumdar ${ }^{\mathrm{j}}$, R.R. McNeil ${ }^{\mathrm{f}}$, S. Mele ${ }^{p}$, L. Merola $^{\mathrm{ab}}{ }$, M. Meschini ${ }^{\circ}$, W.J. Metzger ${ }^{\text {ad }}$, M. von der Mey ${ }^{a}$, D. Migani ${ }^{\mathrm{i}}$, A. Mihul ${ }^{1}$, H. Milcent ${ }^{\mathrm{p}}$, G. Mirabelli ${ }^{\mathrm{a}}$, J. Mnich ${ }^{\mathrm{p}}$, P. Molnar ${ }^{\mathrm{h}}$, B. Monteleoni ${ }^{\circ}$, T. Moulik ${ }^{j}$, G.S. Muanza ${ }^{x}$, F. Muheim ${ }^{r}$, A.J.M. Muijs ${ }^{b}$, S. Nahn ${ }^{n}$, M. Napolitano ${ }^{\text {ab }}$, F. Nessi-Tedaldi ${ }^{\text {au }}$, H. Newman ${ }^{\text {ae }}$, T. Niessen ${ }^{a}$, A. Nippe ${ }^{\text {u }}$, A. Nisati ${ }^{\text {ai }}$, H. Nowak ${ }^{\text {at }}$, Y.D. Oh ${ }^{\text {ao }}$, G. Organtini ${ }^{\text {ai }}$, R. Ostonen ${ }^{\mathrm{t}}$, C. Palomares ${ }^{y}$, D. Pandoulas ${ }^{a}$, S. Paoletti ${ }^{\text {ai, }}$, P. Paolucci ab ${ }^{\text {a }}$ H.K. Park ${ }^{\text {ag }}$, I.H. Park ${ }^{\text {ao }}$, G. Pascale ${ }^{\text {ai }}$, G. Passaleva ${ }^{p}$, S. Patricelli ab, T. Paul ${ }^{\text {k }}$, M. Pauluzzi af, C. Paus ${ }^{\mathrm{p}}$, F. Pauss ${ }^{\text {au }}$, D. Peach ${ }^{\mathrm{p}}$, M. Pedace ${ }^{\text {ai }}$, Y.J. Pei ${ }^{\text {a }}$, S. Pensotti ${ }^{\mathrm{z}}$, D. Perret-Gallix ${ }^{d}$, B. Petersen ${ }^{\text {ad }}$, S. Petrak ${ }^{\text {h }}$, D. Piccolo ${ }^{\text {ab }}$, M. Pieri ${ }^{\circ}$, P.A. Piroué ah , E. Pistolesi z , V. Plyaskin aa ${ }^{\text {a }}$, M. Pohl ${ }^{\text {au }}$, V. Pojidaev aa,o, H. Postema ${ }^{n}$, J. Pothier ${ }^{\mathrm{p}}$, N. Produit ${ }^{\mathrm{r}}$, D. Prokofiev ${ }^{\mathrm{aj}}$, J. Quartieri ak, G. Rahal-Callot ${ }^{\text {au }}$, N. Raja ${ }^{\text {j }, ~ P . G . ~ R a n c o i t a ~}{ }^{z}$, G. Raven ${ }^{\text {al }}$, P. Razis ${ }^{\text {ac }}$, D. Ren ${ }^{\text {au }}$, M. Rescigno ${ }^{\text {ai }}$, S. Reucroft ${ }^{k}$, T. van Rhee ${ }^{\text {aq }}, \mathrm{S}$. Riemann ${ }^{\text {at }}$, K. Riles ${ }^{\text {c }}$, A. Robohm ${ }^{\mathrm{au}}$, J. Rodin ${ }^{\mathrm{ap}}$, B.P. Roe ${ }^{\mathrm{c}}$, L. Romero ${ }^{\mathrm{y}}$, S. Rosier-Lees ${ }^{\mathrm{d}}$, S. Roth ${ }^{\mathrm{a}}$, J.A. Rubio ${ }^{p}$, D. Ruschmeier ${ }^{\mathrm{h}}$, H. Rykaczewski ${ }^{\text {au }}$, S. Sakar ${ }^{\text {ai }}$, J. Salicio ${ }^{\mathrm{p}}$,

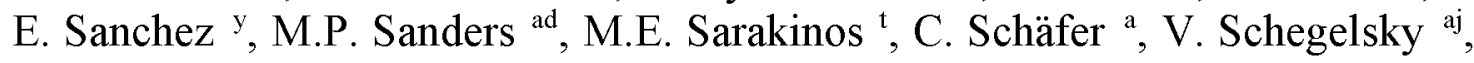
S. Schmidt-Kaerst ${ }^{\text {a }}$, D. Schmitz a , N. Scholz ${ }^{\text {au }}$, H. Schopper av , D.J. Schotanus ${ }^{\text {ad }}$, J. Schwenke a , G. Schwering a , C. Sciacca ${ }^{\text {ab }}$, D. Sciarrino ${ }^{r}$, L. Servoli af, S. Shevchenko ae, N. Shivarov ${ }^{\text {an }}$, V. Shoutko ${ }^{\text {aa }}$, J. Shukla ${ }^{\text {w }}$, E. Shumilov ${ }^{\text {aa }}$, A. Shvorob ${ }^{\text {ae }}$, T. Siedenburg ${ }^{\text {a }}$, D. Son ${ }^{\text {ao }}$, B. Smith ${ }^{\text {ag }}$, P. Spillantini ${ }^{\circ}$, M. Steuer ${ }^{n}$, 


\section{D.P. Stickland ${ }^{\text {ah }}$, A. Stone ${ }^{\mathrm{f}}$, H. Stone ${ }^{\text {ah }}$, B. Stoyanov ${ }^{\text {an }}$, A. Straessner ${ }^{\text {a }}$,} K. Sudhakar ${ }^{\text {j }}$, G. Sultanov ${ }^{\mathrm{q}}$, L.Z. Sun ${ }^{\text {s }}$, H. Suter ${ }^{\text {au }}$, J.D. Swain ${ }^{\mathrm{q}}$, Z. Szillasi ${ }^{\mathrm{a}, 4}$, X.W. Tang ${ }^{g}$, L. Tauscher ${ }^{\text {e, L. Taylor }}{ }^{\mathrm{k}}$, C. Timmermans ${ }^{\text {ad }}$, Samuel C.C. Ting ${ }^{\text {", }}$ S.M. Ting ${ }^{n}$, S.C. Tonwar ${ }^{\mathrm{j}}$, J. Tóth ${ }^{\mathrm{m}}$, C. Tully ${ }^{\text {ah }}$, K.L. Tung ${ }^{\mathrm{g}}$, Y. Uchida ${ }^{\mathrm{n}}$, J. Ulbricht ${ }^{\text {au }}$, E. Valente ${ }^{\text {ai }}$, G. Vesztergombi ${ }^{\mathrm{m}}$, I. Vetlitsky ${ }^{\mathrm{aa}}$, G. Viertel ${ }^{\text {au }}$, S. Villa ${ }^{\mathrm{k}}$, M. Vivargent ${ }^{\mathrm{d}}$, S. Vlachos ${ }^{\mathrm{e}}$, H. Vogel ${ }^{\mathrm{ag}}, \mathrm{H}$. Vogt ${ }^{\text {at }}$, I. Vorobiev ${ }^{\text {p.aa }}$, A.A. Vorobyov aj, A. Vorvolakos ${ }^{\text {ac }}$, M. Wadhwa ${ }^{\text {e }}$, W. Wallraff a , J.C. Wang ${ }^{\text {", }}$, X.L. Wang ${ }^{\text {s }, Z . M . ~ W a n g ~}{ }^{\mathrm{s}}$, A. Weber ${ }^{\mathrm{a}}$, H. Wilkens ${ }^{\text {ad }}$, S.X. Wu ${ }^{\mathrm{n}}$, S. Wynhoff ${ }^{\text {a }}$, L. Xia ${ }^{\text {ae }}$, Z.Z. Xu ${ }^{\text {s, B.Z. Yang }}{ }^{\text {s, C.G. Yang }}{ }^{\mathrm{g}}$, H.J. Yang ${ }^{\mathrm{g}}, \mathrm{M}$. Yang ${ }^{\mathrm{g}}$, J.B. Ye ${ }^{\mathrm{s}}$, S.C. Yeh ${ }^{\text {ax }}$, J.M. You ${ }^{\text {ag }}$, An. Zalite ${ }^{\text {aj }}$, Yu. Zalite ${ }^{\text {aj }}$, P. Zemp ${ }^{\text {au }}$, Y. Zeng ${ }^{\text {a }}$,

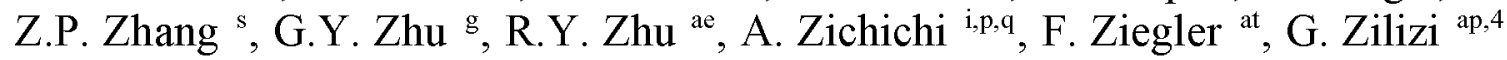

a I. Physikalisches Institut, RWTH, D-52056 Aachen, Germany, and III. Physikalisches Institut, RWTH, D-52056 Aachen, Germany ${ }^{5}$

${ }^{b}$ National Insitiute for High Energy Physics, NIKHEF, and University of Amsterdam, NL-1009 DB Amsterdam, The Netherlands

c University of Michigan, Ann Arbor, MI 48109, USA

${ }^{\mathrm{d}}$ Laboratoire d'Annecy-le-Vieux de Physique des Particules, LAPP, IN2P3-CNRS, BP 110, F-7494I Annecy-le-Vieux CEDEX, France

'Institute of Physics, University of Basel, CH-4056 Basel, Switzerland

${ }^{\mathrm{f}}$ Louisiana State University, Baton Rouge, LA 70803, USA

${ }^{g}$ Institute of High Energy Physics, IHEP, 100039 Beijing, China ${ }^{6}$

${ }^{\mathrm{h}}$ Humboldt University, D-10099 Berlin, Germany ${ }^{5}$

${ }^{\mathrm{i}}$ University of Bologna and INFN-Sezione di Bologna, I-40126 Bologna, Italy

${ }^{\mathrm{j}}$ Tata Institute of Fundamental Research, Bombay 400005 , India

${ }^{\mathrm{k}}$ Northeastern University, Boston, MA 02115, USA

${ }^{1}$ Institute of Atomic Physics and University of Bucharest, R-76900 Bucharest, Romania

${ }^{\mathrm{m}}$ Central Research Institute for Physics of the Hungarian Academy of Sciences, H-1525 Budapest 114, Hungary ${ }^{7}$

${ }^{\mathrm{n}}$ Massachusetts Institute of Technology, Cambridge, MA 02139, USA

INFN Sezione di Firenze and University of Florence, I-50125 Florence, Italy

${ }^{\mathrm{p}}$ European Laboratory for Particle Physics, CERN, CH-1211 Geneva 23, Switzerland

${ }^{\mathrm{q}}$ World Laboratory, FBLJA Project, $\mathrm{CH}-1211$ Geneva 23, Switzerland

${ }^{\mathrm{r}}$ University of Geneva, CH-1211 Geneva 4, Switzerland

s Chinese University of Science and Technology, USTC, Hefei, Anhui 230 029, China ${ }^{6}$

${ }^{t}$ SEFT, Research Institute for High Energy Physics, P.O. Box 9, SF-00014 Helsinki, Finland

"University of Lausanne, CH-1015 Lausanne, Switzerland

"INFN-Sezione di Lecce and Universitá Degli Studi di Lecce, I-73100 Lecce, Italy

${ }^{w}$ Los Alamos National Laboratory, Los Alamos, NM 87544, USA

${ }^{\mathrm{x}}$ Institut de Physique Nucleaire de Lyon, IN2P3-CNRS, Université Claude Bernard, F-69622 Villeurbanne, France

${ }^{y}$ Centro de Investigaciones Energéticas, Medioambientales y Tecnologícas, CIEMAT, E-28040 Madrid, Spain ${ }^{8}$

${ }^{\mathrm{z}}$ INFN-Sezione di Milano, I-20133 Milan, Italy

aa Institute of Theoretical and Experimental Physics, ITEP, Moscow, Russia

${ }^{\text {ab }}$ INFN-Sezione di Napoli and University of Naples, I-80125 Naples, Italy

ac Department of Natural Sciences, University of Cyprus, Nicosia, Cyprus

ad University of Nijmegen and NIKHEF, NL-6525 ED Nijmegen, The Netherlands

${ }^{\text {ae }}$ California Institute of Technology, Pasadena, CA 91125, USA

${ }^{\text {af }}$ INFN-Sezione di Perugia and Universitó Degli Studi di Perugia, I-06100 Perugia, Italy

ag Carnegie Mellon University, Pittsburgh, PA 15213, USA

ah Princeton University, Princeton, NJ 08544, USA

ai INFN-Sezione di Roma and University of Rome, "La Sapienza", I-00185 Rome, Italy

aj Nuclear Physics Institute, St. Petersburg, Russia

ak University and INFN, Salerno, I-84100 Salerno, Italy

al University of California, San Diego, CA 92093, USA

am Dept. de Fisica de Particulas Elementales, Univ. de Santiago, E-15706 Santiago de Compostela, Spain

an Bulgarian Academy of Sciences, Central Lab. of Mechatronics and Instrumentation, BU-1113 Sofia, Bulgaria 


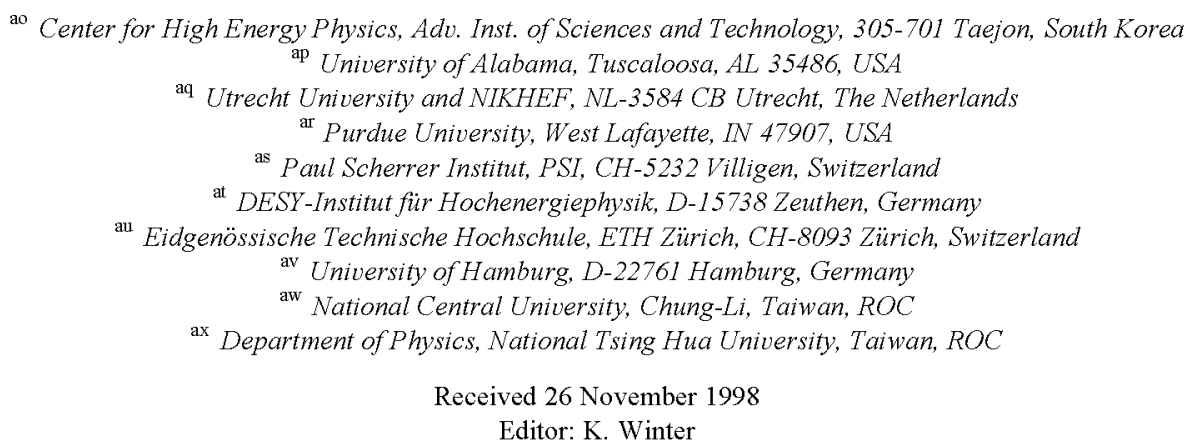

\begin{abstract}
The cross section of charm production in $\gamma \gamma$ collisions $\sigma\left(\mathrm{e}^{+} \mathrm{e}^{-} \rightarrow \mathrm{e}^{+} \mathrm{e}^{-} \mathrm{c} \overline{\mathrm{c} X}\right)$ is measured at LEP with the L3 detector at centre-of-mass energies from $91 \mathrm{GeV}$ to $183 \mathrm{GeV}$. Charmed hadrons are identified by electrons and muons from semileptonic decays. The direct process $\gamma \gamma \rightarrow \mathrm{c} \overline{\mathrm{c}}$ is found to be insufficient to describe the data. The measured cross section values and event distributions require contributions from resolved processes, which are sensitive to the gluon density in the photon. (C) 1999 Published by Elsevier Science B.V. All rights reserved.
\end{abstract}

\section{Introduction}

The production of heavy quarks in two-photon collisions consists mainly of charm quarks. Because of their smaller electric charge and larger mass, the production of $b$-quarks is expected to be suppressed by more than two orders of magnitude relative to the production of charm quarks [1]. The measurement of charm production in two-photon collisions provides a good test of QCD because the large physical scale set by the charm quark mass makes the perturbative

\footnotetext{
${ }^{1}$ Also supported by CONICET and Universidad Nacional de La Plata, CC 67, 1900 La Plata, Argentina.

${ }^{2}$ Also supported by Panjab University, Chandigarh-160014, India.

${ }^{3}$ Supported by Deutscher Akademischer Austauschdienst.

${ }^{4}$ Also supported by the Hungarian OTKA fund under contract numbers $\mathrm{T} 22238$ and $\mathrm{T} 026178$.

${ }^{5}$ Supported by the German Bundesministerium für Bildung, Wissenschaft, Forschung und Technologie.

${ }^{6}$ Supported by the National Natural Science Foundation of China.

${ }^{7}$ Supported by the Hungarian OTKA fund under contract numbers T019181, F023259 and T024011.

${ }^{8}$ Supported also by the Comisión Interministerial de Ciencia y Tecnología.
}

calculations reliable. At LEP energies, the direct and resolved processes (Fig. 1) are predicted to give comparable contributions to the cross section [1]. The contributions to charm production from soft processes described by the Vector Dominance Model (VDM) and from doubly resolved processes are expected to be small. The resolved photon cross section is dominated by the photon-gluon fusion diagram $\gamma \mathrm{g} \rightarrow \mathrm{c} \overline{\mathrm{c}}$. The production rate of charm quarks in two-photon collisions depends on the charm quark mass and on the gluon density in the photon. Measurements of charm production in two-photon collisions were done at PEP, PETRA, TRISTAN and LEP [2-8], where charm quarks were identified by charged $\mathrm{D}^{*}$ mesons, inclusive electrons or muons and $\mathrm{K}_{\mathrm{S}}^{0}$ mesons.

In this paper we present the result of the measurement of the $\mathrm{e}^{+} \mathrm{e}^{-} \rightarrow \mathrm{e}^{+} \mathrm{e}^{-} \mathrm{c} \overline{\mathrm{c} X}$ cross section by the $\mathrm{L} 3$ experiment at $\sqrt{\mathrm{s}}=91 \mathrm{GeV}$ and at energies above the $Z$ mass. We identify charm quarks by tagging electrons ${ }^{9}$ and muons from semileptonic charm decays. The data correspond to a total integrated luminosity of $\mathscr{L}=165 \mathrm{pb}^{-1}$. For the first

\footnotetext{
${ }^{9}$ Electron stands for electron or positron throughout this paper.
} 


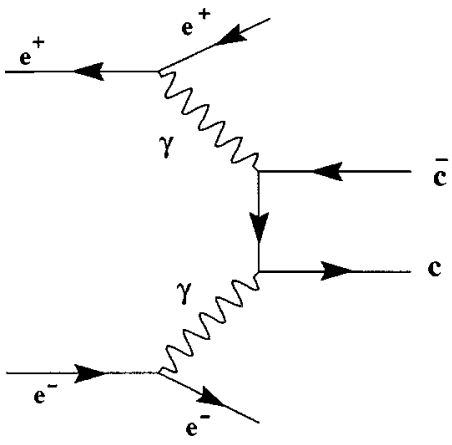

Direct

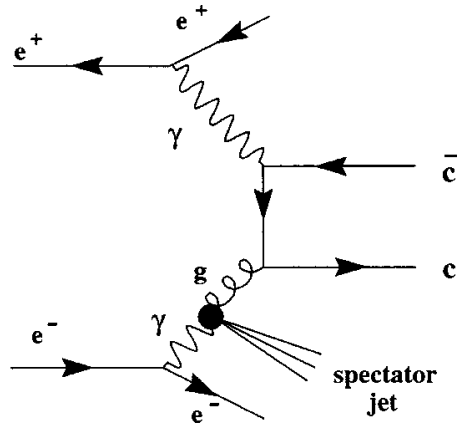

Single Resolved

Fig. 1. Diagrams contributing to charm production in $\gamma \gamma$ collisions at LEP. Contributions from other processes, double resolved process and VDM, are predicted to be small at LEP energies [1].

time the charm production cross section is measured at more than one energy in a single experiment.

\section{Selection of hadronic two-photon events}

The L3 detector has been described in detail in Ref. [9]. The event selection is done in two steps. The first one selects hadronic final states produced in two-photon collisions, and the second identifies a charm quark. Hadronic two-photon events are selected by cuts on the number of tracks, the visible energy and the visible mass. The visible energy, $E_{v i s}$, is the sum of the energies measured in the calorimeters and that of the muons measured in the muon spectrometer. The visible mass, $\mathrm{W}_{\mathrm{vis}}$, of the event is calculated from the four-momentum vectors of the measured calorimetric clusters. All particles are considered to be pions except for electromagnetic (EM) clusters identified as photons. We require at least five tracks in each event; the visible energy has to be less than $0.38 \sqrt{s}$ and the visible mass has to be greater than $3 \mathrm{GeV}$. As one can see in Fig. $2 \mathrm{a}$ and Fig. $2 b$, the cut on $E_{v i s}$ separates the two-photon from annihilation processes which are characterized by high visible energy. The data are reasonably well described by the simulation except for the normalization of the $\gamma \gamma \rightarrow$ hadrons contribution, while both the shape and the normalization of the annihilation background are well reproduced by the Monte Carlo. The background from $\mathrm{e}^{+} \mathrm{e}^{-} \rightarrow \mathrm{e}^{+} \mathrm{e}^{-} \tau^{+} \tau^{-}$and $\mathrm{e}^{+} \mathrm{e}^{-} \rightarrow \tau^{+} \tau^{-}$events is highly suppressed by the five tracks requirement.
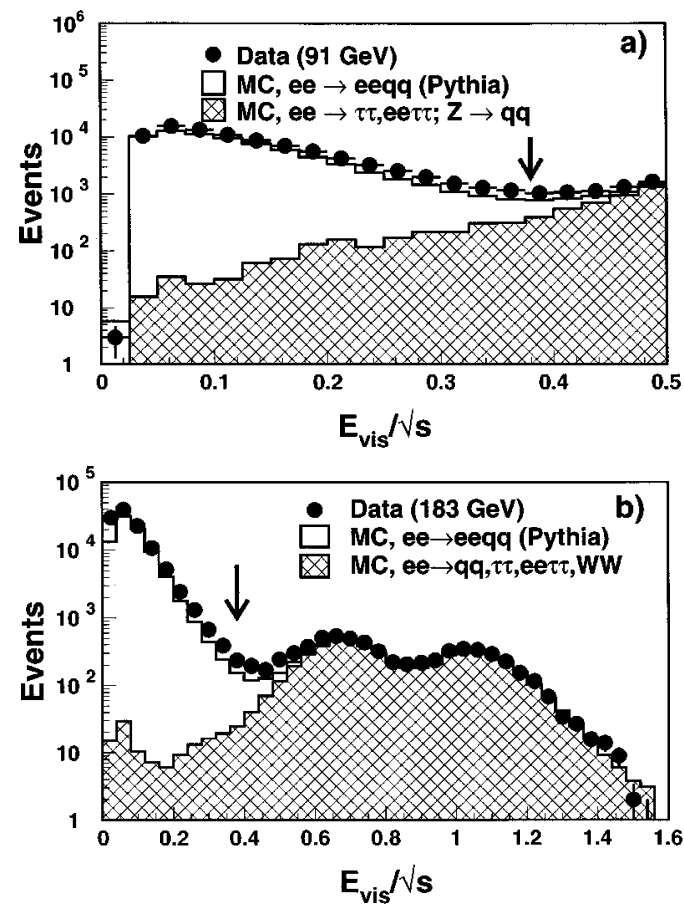

Fig. 2. Total visible energy in the data at a) $\sqrt{\mathrm{s}}=91 \mathrm{GeV}$ and at b) $\sqrt{\mathrm{s}}=183 \mathrm{GeV}$. Also shown are the Monte Carlo predictions for two-photon hadron production and the main backgrounds. A cut at $\mathrm{E}_{\mathrm{vis}}<0.38 \sqrt{\mathrm{s}}$ removes most of the background coming from the annihilation channels. Because of the large $Z$ decay background at $\sqrt{\mathrm{s}}=91 \mathrm{GeV}$ only the interval $\mathrm{E}_{\mathrm{yis}}<0.5 \sqrt{\mathrm{s}}$ is shown in a). 
Table 1

Data samples collected by L3 from 1994 to 1997 at $\sqrt{\mathrm{s}}=91-183 \mathrm{GeV}$ and the corresponding integrated luminosities $\mathscr{L}$. The number of selected hadronic events is given together with the number of events selected with the electron or muon tag. The inclusive lepton cross section $\Delta \sigma$ is calculated after subtraction of annihilation and $\mathrm{e}^{+} \mathrm{e}^{-} \rightarrow \mathrm{e}^{+} \mathrm{e}^{-} \tau^{+} \tau^{-}$background in the polar angle region $|\cos \theta|<0.9$, for a momentum greater than $0.6(2.0) \mathrm{GeV}$ for electrons (muons) and with $\mathrm{W} \gamma \gamma>3 \mathrm{GeV}$. The first error is statistical and the second one is systematic

\begin{tabular}{|c|c|c|c|c|c|c|}
\hline $\begin{array}{c}\sqrt{s} \\
{[\mathrm{GeV}]}\end{array}$ & $\begin{array}{c}\mathscr{L} \\
{\left[\mathrm{pb}^{-1}\right]}\end{array}$ & $\begin{array}{c}\text { Hadronic } \\
\text { Events }\end{array}$ & \multicolumn{2}{|c|}{ Electron Tag } & \multicolumn{2}{|c|}{ Muon Tag } \\
\hline 91 & 79.8 & 93204 & 282 & $25.9 \pm 2.1 \pm 3.7$ & 57 & $1.64 \pm 0.30 \pm 0.08$ \\
\hline $161-172$ & 21.2 & 44444 & 156 & $64.6 \pm 6.3 \pm 5.9$ & 16 & $2.31 \pm 0.63 \pm 0.12$ \\
\hline 183 & 52.2 & 116760 & 433 & $77.8 \pm 4.4 \pm 5.0$ & 52 & $3.33 \pm 0.49 \pm 0.16$ \\
\hline
\end{tabular}

The analysis is limited to untagged events with small photon virtuality. Events are excluded when the most energetic cluster in the L3 luminosity monitor has an energy greater than $0.4 \mathrm{E}_{\text {beam. }}$. Thus the interacting photons are quasi-real: $\left\langle\mathrm{Q}^{2}\right\rangle \cong 0.1 \mathrm{GeV}^{2}$, where $-Q^{2}$ is the invariant mass squared of the virtual photon.

The numbers of events selected at different energies are given in Table 1. Background sources are the two-photon process $\mathrm{e}^{+} \mathrm{e}^{-} \rightarrow \mathrm{e}^{+} \mathrm{e}^{-} \tau^{+} \tau^{-}$simulated with the JAMVG [10] Monte Carlo generator, and the annihilation processes $\mathrm{e}^{+} \mathrm{e}^{-} \rightarrow \mathrm{Z} / \gamma \rightarrow \mathrm{q} \overline{\mathrm{q}}$, simulated with JETSET $7.3[11]$ at $\sqrt{\mathrm{s}}=91 \mathrm{GeV}$ and with PYTHIA 5.7 [12] at energies above the $Z$ mass. The process $\mathrm{e}^{+} \mathrm{e}^{-} \rightarrow \tau^{+} \tau^{-}$is simulated with $\mathrm{KO}$ RALZ [13], and, at higher energies, $\mathrm{e}^{+} \mathrm{e}^{-} \rightarrow \mathrm{W}^{+} \mathrm{W}^{-}$ with KORALW [14]. After the hadron selection, the background from the annihilation processes and the two-photon production of tau pairs is about $2 \%$ at $\sqrt{\mathrm{s}}=91 \mathrm{GeV}$ and is below $1 \%$ at higher energies. The background at $\sqrt{\mathrm{s}}=91 \mathrm{GeV}$ is dominated by the $\mathrm{e}^{+} \mathrm{e}^{-} \rightarrow \mathrm{Z} \rightarrow \mathrm{q} \overline{\mathrm{q}}$ process.

The PYTHIA Monte Carlo simulates two-photon events according to the current knowledge of hadronic interactions obtained by $p p$ and $\gamma p$ studies [12]. The two-photon processes are generated with massless $\left(\mathrm{m}_{\mathrm{q}}=0\right)$ matrix elements [15]. The resolved process uses the SaS1d photon structure function [16]. We have implemented the two-photon luminosity function in the equivalent photon approximation (EPA) which has a cutoff: $\mathrm{Q}^{2}<\mathrm{m}_{\rho}^{2}$ [17].

The detector simulation was performed by a GEANT-based description of the L3 detector [18]. The Monte Carlo events are reconstructed in the same way as the data.

\section{Electron selection}

To identify charm-quark production, we search for an electron with momentum greater than $0.6 \mathrm{GeV}$
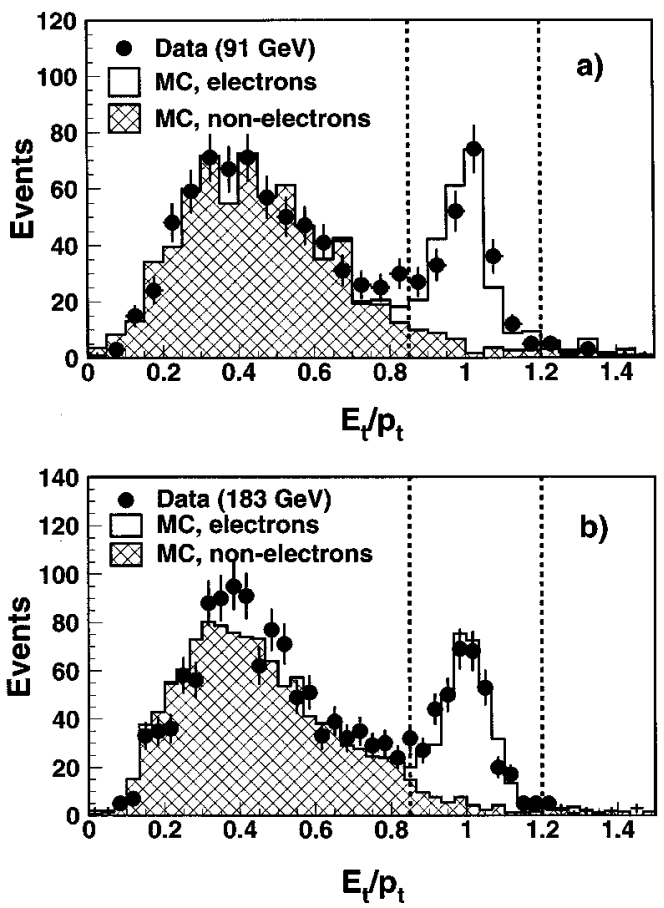

Fig. 3. The ratio, $E_{t} / p_{t}$, of the transverse energy measured by the electromagnetic calorimeter and the transverse momentum of the track for a) $\sqrt{\mathrm{s}}=91 \mathrm{GeV}$ and b) $\sqrt{\mathrm{s}}=183 \mathrm{GeV}$. A clear electron signal is observed at the expected value of $E_{t} / p_{t}=1$. The window, defined by the dashed lines, of $0.85<\mathrm{E}_{\mathrm{t}} / \mathrm{p}_{\mathrm{t}}<1.2$ indicates the selected electron candidates. 
in the polar angle range $|\cos \theta|<0.9$. Electron candidates are selected as follows:

- The EM cluster matches to a track; the difference between the azimuthal angles estimated from the shower barycentre and from the track impact point at the calorimeter must be smaller than 20 mrad.

- To confirm that a shower in the EM calorimeter is created by an electron, the distribution of energies measured in the crystals of the calorimeter are compared to that of an EM cluster using a $\chi^{2}$ test.

- The cluster must also satisfy the condition 0.85 $<\mathrm{E}_{\mathrm{t}} / \mathrm{p}_{\mathrm{t}}<1.20$ (Fig. Fig. 3), where $\mathrm{E}_{\mathrm{t}}$ is the projection of the energy of the cluster on the $r-\phi$ plane and $p_{t}$ is the transverse momentum of the track as measured in the central tracker. This condition rejects more than $95 \%$ of the hadrons (mainly pions) while keeping more than $90 \%$ of the electrons. The average resolutions for the selected electron candidates are $4.6 \%$ on $p_{t}$ and $3.3 \%$ on $\mathrm{E}_{\mathrm{t}}$.

- The distance of closest approach of the track to the average position of the $\mathrm{e}^{+} \mathrm{e}^{-}$collision point in the $r-\phi$ plane must be less than $0.5 \mathrm{~mm}$. This cut rejects $71 \%$ of electron candidates from $\gamma$ conversions while keeping more than $88 \%$ of signal electrons.

The inclusive electron cross section in the fiducial volume of $|\cos \theta|<0.9$, with a momentum greater than $0.6 \mathrm{GeV}$ and $\mathrm{W}_{\gamma \gamma}>3 \mathrm{GeV}$ is calculated as:

$\Delta \sigma_{\mathrm{e}}=\frac{\left[\left(N_{\mathrm{obs}}^{\mathrm{lept}}-N_{\mathrm{bkg}}^{\mathrm{lept}}\right) \mathrm{P}_{\mathrm{e}}\right]-N_{\text {conv }}}{\mathscr{L} \epsilon_{\mathrm{trig}} \epsilon_{\mathrm{e}}}$

The variables are defined as follows:

- $N_{\mathrm{obs}}^{\text {lept }}$ is the number of events in the data after the final electron selection.

- $\epsilon_{\text {trig }}$ is the trigger efficiency which is determined from the data using a set of independent triggers.

- $N_{\mathrm{bkg}}^{\text {lept }}$ is the number of background events estimated from Monte Carlo which do not originate from two-photon hadronic interactions.

- $N_{\text {conv }}$ is the estimated number of $\gamma$-conversion electrons. This background comprises about $22 \%$ of the selected electron sample.

- $\mathscr{L}$ is the total integrated luminosity.
- $\epsilon_{\mathrm{e}}$, the electron selection efficiency, is the fraction of electrons generated within $|\cos \theta|<0.9$, with a momentum greater than $0.6 \mathrm{GeV}$ and $\mathrm{W}_{\gamma \gamma}>3$ $\mathrm{GeV}$ which remains after final selection.

- $P_{e}$ is the electron purity in the selected sample.

The number of observed events is given in Table 1 . The trigger efficiencies range from $87 \pm 1 \%$ at $\sqrt{\mathrm{s}}=$ $91 \mathrm{GeV}$ to $79 \pm 1 \%$ at $\sqrt{\mathrm{s}}=183 \mathrm{GeV}$. The background from the annihilation processes and the twophoton production of tau pairs is $10 \%$ at $\sqrt{\mathrm{s}}=91$ $\mathrm{GeV}$ and is about $1 \%$ at higher energies. We assume that this background has the same trigger efficiencies as the hadronic two-photon signal events. The efficiency after hadronic selection is $43 \%$. The electron selection reduces the electron efficiency to about $10 \%$ (mostly due to electromagnetic shower shape requirement). The electron purity is $85 \%$. Both fractions are estimated using the PYTHIA Monte Carlo. The Monte Carlo includes electrons from $\pi^{0}$ Dalitz decays, such electrons give a contribution of $12.7 \%$ to the electron sample.

The measured cross sections, $\Delta \sigma_{\mathrm{e}}$, are given in Table 1. We observe an increase of the cross section with increasing beam energy. The dominant systematic errors are from the uncertainty on background subtraction, selection efficiency and cut variation. In Fig. 4 , the differential cross section at $183 \mathrm{GeV}$ is plotted as a function of the transverse momentum of the electron. The prediction (normalized to the number of data events) from the PYTHIA Monte Carlo

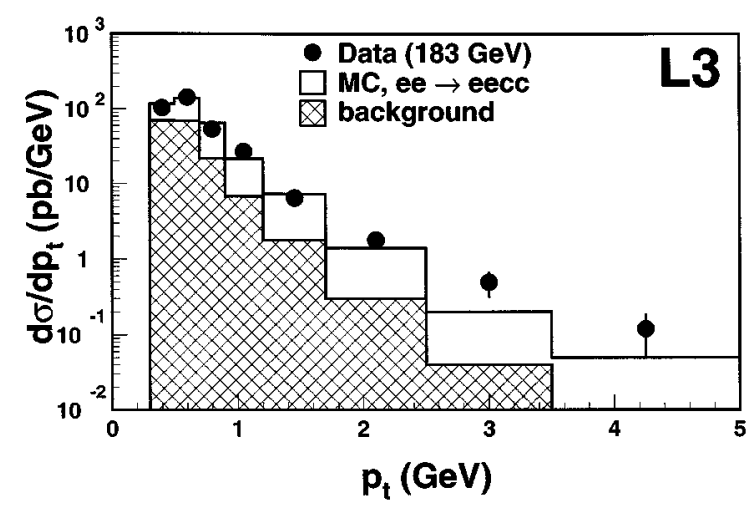

Fig. 4. The differential cross section for inclusive electrons at $\sqrt{\mathrm{s}}=183 \mathrm{GeV}$ as a function of the electron transverse momentum. The data are compared to the total PYTHIA Monte Carlo predictions scaled to the observed cross section. The shaded area shows the non-charm two-photon events background. The cross section is given for the kinematic range defined in the text. 
for inclusive charm production and background is also shown. The shape of the distribution is in agreement with the prediction. Leptons from semileptonic decays of charm quarks are on average more energetic than leptons from non-charm twophoton processes, therefore the charm purity increases with the transverse momentum. The absolute prediction for the number of events is $10 \%(60 \%)$ too small at $\sqrt{\mathrm{s}}=91 \mathrm{GeV}(183 \mathrm{GeV})$, this difference can be attributed to the fact that the PYTHIA Monte Carlo contains only leading order calculations.

\section{Muon selection}

The muon candidate is required to have a momentum greater than $2 \mathrm{GeV}$ because only such muons can penetrate the calorimeters and reach the muon chambers. In order to suppress the contribution from the annihilation processes, we require the muon momentum to be less than $0.2 \mathrm{E}_{\text {beam }}$. The angular acceptance is limited to $|\cos \theta|<0.9$.

After all cuts are applied, 57, 16 and 52 events remain at the centre-of-mass energies $\sqrt{\mathrm{s}}=91 \mathrm{GeV}$, $167 \mathrm{GeV}$ and $183 \mathrm{GeV}$, respectively. The inclusive muon cross section is calculated for $|\cos \theta|<0.9$, a momentum greater than $2 \mathrm{GeV}$ and $\mathrm{W}_{\gamma \gamma}>3 \mathrm{GeV}$. The muon selection efficiency, $\epsilon_{\mu}$, is estimated to be $33 \%$; the muon purity, $\mathrm{P}_{\mu}$, is $100 \%$. The trigger efficiency is higher by a factor 1.08 than in the case of the electron selection due to the higher momentum cut. The measured cross sections, $\Delta \sigma_{\mu}$, are given in Table 1. The background from annihilation processes and two-photon production of tau pairs is $24 \%$ at $\sqrt{\mathrm{s}}=91 \mathrm{GeV}$ and about $5 \%$ at higher energies. Systematic errors arise from the uncertainty on background subtraction, selection efficiency, trigger efficiency and cut variation. The statistical error is dominant for this measurement and amounts to about $16 \%$ at $\sqrt{\mathrm{s}}=91 \mathrm{GeV}$ and $183 \mathrm{GeV}$.

\section{Results}

The total cross section of inclusive charm production is calculated from the following equation:

$$
\sigma=\frac{\left(N_{\mathrm{obs}}^{\mathrm{lept}}-N_{\mathrm{bkg}}^{\mathrm{lept}}\right) \pi_{\mathrm{c}}}{\mathscr{L} \epsilon_{\mathrm{trig}} \epsilon_{\mathrm{c}}^{\prime}} .
$$

The charm selection efficiency, $\epsilon_{\mathrm{c}}^{\prime}$, is the fraction of charm events selected by the lepton tag analysis relative to the events generated in the full phase space. The charm purity, $\pi_{c}$, is defined as:

$$
\pi_{\mathrm{c}}=\frac{N_{\mathrm{c}}^{\text {lept }}}{N_{\mathrm{c}}^{\text {lept }}+N_{\mathrm{nc}}^{\text {lept }}} .
$$

In order to be less dependent on the Monte Carlo flavour composition (charm to non-charm fraction), the charm purity can be rewritten as:

$$
\pi_{\mathrm{c}}=\left(1-\frac{\epsilon_{\mathrm{nc}}}{\epsilon_{\mathrm{d}}}\right) /\left(1-\frac{\epsilon_{\mathrm{nc}}}{\epsilon_{\mathrm{c}}}\right),
$$

where the $\epsilon_{\mathrm{c}}\left(\epsilon_{\mathrm{nc}}\right)$ is the fraction of charm $N_{\mathrm{c}}^{\text {lept }}$ (non-charm $N_{\text {nc }}^{\text {lept }}$ ) events, accepted by the final selection from the charm (non-charm) events obtained after the hadronic selection. The quantity $\epsilon_{d}$ is defined by the relation:

$\epsilon_{\mathrm{d}}=\frac{N_{\mathrm{c}}^{\text {lept }}+N_{\mathrm{nc}}^{\text {lept }}}{N_{\mathrm{c}}^{\text {had }}+N_{\mathrm{nc}}^{\text {had }}}=\frac{N_{\mathrm{obs}}^{\text {lept }}-N_{\mathrm{bkg}}^{\text {lept }}}{N_{\mathrm{obs}}^{\text {had }}-N_{\mathrm{bkg}}^{\text {had }}}$

and can thus be determined directly from the data. Eq. (4) is obtained by noticing that the total number of selected hadronic events $N_{\mathrm{c}}^{\text {had }}+N_{\mathrm{nc}}^{\text {had }}$ can be expressed as:

$$
\frac{N_{\mathrm{c}}^{\text {lept }}+N_{\mathrm{nc}}^{\text {lept }}}{\boldsymbol{\epsilon}_{\mathrm{d}}}=\frac{N_{\mathrm{c}}^{\text {lept }}}{\boldsymbol{\epsilon}_{\mathrm{c}}}+\frac{N_{\mathrm{nc}}^{\text {lept }}}{\boldsymbol{\epsilon}_{\mathrm{nc}}} .
$$

This method of deriving the charm cross section is insensitive to the absolute normalization of the charm and background Monte Carlo, but still depends on the ratio of direct to resolved process in the signal Monte Carlo, as discussed below.

The charm purity is about 0.60 for electrons and about 0.65 for muons ${ }^{10}$. The purity calculated with the use of $\epsilon_{\mathrm{d}}$ from the data gives on average a value about $10 \%$ higher than the estimate using only the Monte Carlo. For the electron sample, the charm selection efficiency $\epsilon_{\mathrm{c}}^{\prime}$ increases from $0.44 \%$ at

\footnotetext{
${ }^{10}$ Besides charm quark semileptonic decays other sources of electrons and muons are the decays $\tau^{+} \rightarrow l^{+} \nu_{l} \bar{\nu}_{\tau}, \pi^{+} \rightarrow \mu^{+} \nu_{\mu}, \pi^{0}$ $\rightarrow e^{+} e^{-} \gamma, K^{+} \rightarrow \mu^{+} \nu_{\mu}, l^{+} \pi^{0} \nu_{l}, K_{L}^{0} \rightarrow l^{+} \pi^{-} \nu_{l}, \ldots$
} 
Table 2

Total cross section values for the process $\mathrm{e}^{+} \mathrm{e}^{-} \rightarrow \mathrm{e}^{+} \mathrm{e}^{-} \mathrm{c} \overline{\mathrm{c} X}$ at four different energies using electron and muon identification. The statistical and systematic uncertainties are also given. In the last column, the data from both leptons are combined

\begin{tabular}{cccc}
\hline $\begin{array}{c}\sqrt{\mathrm{s}} \\
{[\mathrm{GeV}]}\end{array}$ & $\begin{array}{c}\text { Electron tag } \\
\boldsymbol{\sigma}[\mathrm{nb}]\end{array}$ & $\begin{array}{c}\text { Muon tag } \\
\boldsymbol{\sigma}[\mathrm{nb}]\end{array}$ & $\begin{array}{c}\text { Combined } \\
\boldsymbol{\sigma}[\mathrm{nb}]\end{array}$ \\
\hline 91 & $0.44 \pm 0.06 \pm 0.08$ & $0.60 \pm 0.17 \pm 0.16$ & $0.46 \pm 0.06 \pm 0.07$ \\
133 & $1.36 \pm 0.24 \pm 0.18$ & - & $1.36 \pm 0.24 \pm 0.18$ \\
167 & $1.01 \pm 0.15 \pm 0.11$ & $0.58 \pm 0.36 \pm 0.19$ & $0.94 \pm 0.14 \pm 0.10$ \\
183 & $1.29 \pm 0.11 \pm 0.12$ & $1.26 \pm 0.33 \pm 0.25$ & $1.29 \pm 0.10 \pm 0.11$ \\
\hline
\end{tabular}

$\sqrt{\mathrm{s}}=91 \mathrm{GeV}$ to $0.53 \%$ at $\sqrt{\mathrm{s}}=183 \mathrm{GeV}$. This efficiency is much lower for the muon sample, about $0.05 \%$ at all energies, due to the higher momentum cut.

The cross sections are given in Table 2 with statistical and systematic uncertainties. Systematic errors arise from the uncertainty on background subtraction, selection efficiencies, trigger efficiency, cut variation and charm semileptonic branching ratio. The average charm semileptonic branching ratio used in the simulation is 0.098 [19]. The dominant systematic errors for the electron sample are from the cut variation (from $9.5 \%$ at $\sqrt{\mathrm{s}}=91 \mathrm{GeV}$ to $6.5 \%$ at $\sqrt{\mathrm{s}}=183 \mathrm{GeV}$ ) and selection efficiencies (from $12.6 \%$ at $\sqrt{\mathrm{s}}=91 \mathrm{GeV}$ to $5.9 \%$ at $\sqrt{\mathrm{s}}=183 \mathrm{GeV}$ ). The dominant systematic error for the muon sample comes from selection efficiencies (from $24.2 \%$ at $\sqrt{\mathrm{s}}=91 \mathrm{GeV}$ to $18.4 \%$ at $\sqrt{\mathrm{s}}=183 \mathrm{GeV})$.

The charm production cross sections are obtained with the PYTHIA Monte Carlo using a massless quark matrix element calculation. The effect of the use of massive matrix elements is tested by using the PYTHIA Monte Carlo events generated with the charm mass $m_{c}=1.6 \mathrm{GeV}$. Since the cross section values are dependent on the ratio of the charm purity to the charm selection efficiency, $\pi_{c} / \epsilon_{c}^{\prime}$, we compare the value of this ratio using the massless matrix elements to the ratios obtained from the massive matrix elements approach. Within statistics they are the same. The change of the direct to resolved process ratio in the signal Monte Carlo by a factor 1.2 (1.4) results in a change of the charm cross section by $3.4 \%(6 \%)$ for electrons and has negligible effect for muons.

The PYTHIA Monte Carlo is the only generator available which includes all hadronic two-photon processes relevant in this analysis. The QED JAMVG program generates only the direct process. To better understand possible systematics due to the different models, we have compared the values of $\pi_{c} / \epsilon_{c}^{\prime}$ for the direct process as given by PYTHIA and by JAMVG. There is agreement within $10 \%$ which is comparable with the statistical uncertainty. However, for low momenta as seen in the case of the electron selection, the value of $\pi_{c} / \epsilon_{c}^{\prime}$ for the direct process is two times higher than that for the resolved process.

The most sensitive distributions where predictions for direct and resolved processes are different are found to be the visible mass, the track multiplicity, the transverse momentum of the lepton and the energy flow spectra [20]. A comparison of the visible mass distribution in data with the expectations of the direct and all two-photon processes for the high statistics electron sample at $\sqrt{s}=183 \mathrm{GeV}$ is given in Fig. 5. The direct process decreases more quickly

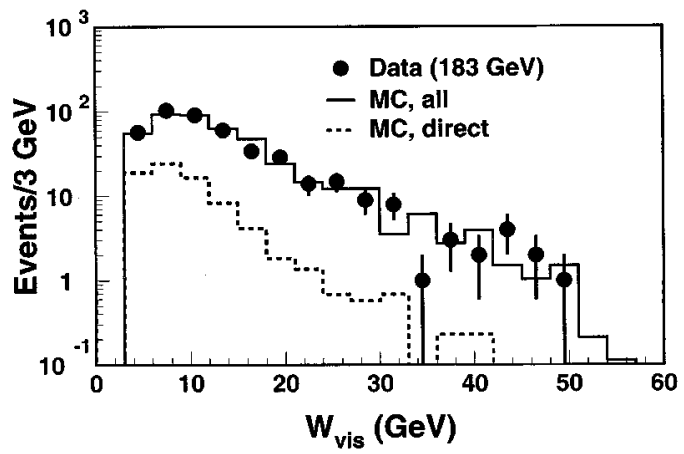

Fig. 5. The visible mass spectrum for the inclusive electron data at $\sqrt{\mathrm{s}}=183 \mathrm{GeV}$ compared to PYTHIA events generated with massless matrix elements. The Monte Carlo spectrum with all contributions is normalized to the same number of events as the data. The dashed histogram shows the contribution from the direct process. 
than the resolved process with increasing visible mass. In Fig. 6, we plot the energy flow as a function of the pseudorapidity, $\eta=-\ln \left(\tan \left(\frac{\theta}{2}\right)\right)$, where $\theta$ is the polar angle of the particle. A clear difference in shape can be seen between the distributions for the direct and resolved processes for $|\eta|>1$, where the direct process alone is insufficient to describe the data.

The total inclusive charm production cross sections are plotted in Fig. 7 together with previous measurements [2-8]. For the purpose of comparison, the published results of different experiments were extrapolated to the total charm cross sections using the procedure of Ref. [21]. The data are compared to the predictions of Ref. [1]. The dashed line corresponds to the direct process, NLO QCD calculations, while the solid line shows the QCD prediction for the sum of direct and resolved processes calculated to NLO accuracy. The direct process depends upon the heavy-quark mass and the QCD coupling constant. The prediction is calculated using a charm mass of $1.3 \mathrm{GeV}$; the open charm threshold energy is set to $3.8 \mathrm{GeV}$. The theory prediction for the resolved process was calculated with the parton density function of Glück-Reya-Vogt [22]. Using the Drees-Grassie [23] parametrization for the photon parton density results in a decrease of the cross section of $9 \%$ for $m_{c}=1.3 \mathrm{GeV}$ and of $3 \%$ for $\mathrm{m}_{\mathrm{c}}=1.7 \mathrm{GeV}$. The renormalization scale was chosen to be the charm mass. A change in the renormalization scale from $m_{c}$ to $2 m_{c}$ decreases the QCD

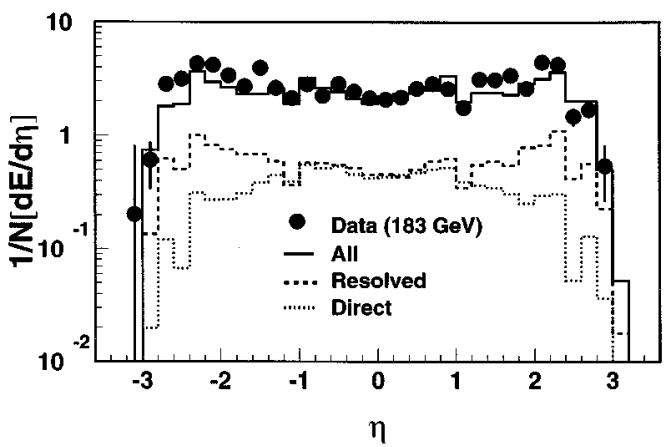

Fig. 6. Energy flow as a function of pseudorapidity $\eta$. The data are compared to the PYTHIA prediction with all contributions (solid histogram) and to the direct and single-resolved processes separately (dotted and dashed histograms, respectively).

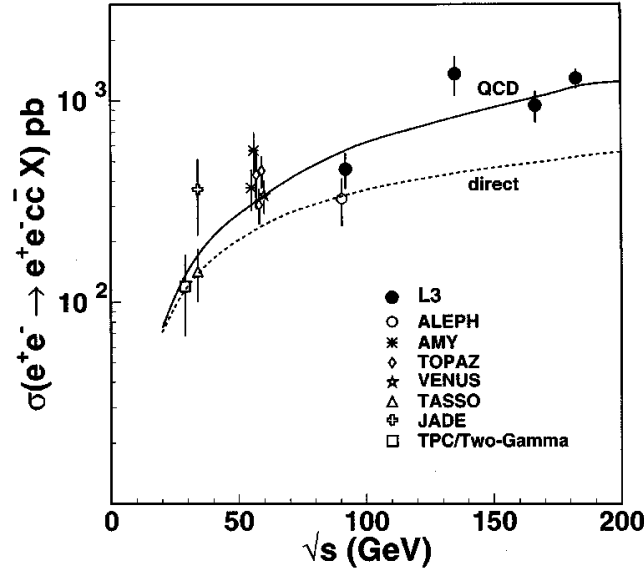

Fig. 7. The charm production cross section in two-photon collisions. The L3 data from both the electron and the muon events are combined. The statistical and systematic errors are added in quadrature. The dashed line corresponds to the direct process prediction while the solid line shows the QCD prediction for the sum of the direct and the resolved processes calculated to NLO accuracy [1]. The prediction corresponds to a calculation for a charm quark mass of $1.3 \mathrm{GeV}$, the parton density function of Glück-Reya-Vogt [22] and the renormalization scale was chosen to be the charm quark mass. Points at $\sqrt{\mathrm{s}}=58 \mathrm{GeV}$ and $\sqrt{\mathrm{s}}=91$ $\mathrm{GeV}$ energies are artificially separated for clear visibility

prediction by $30 \%(15 \%)$ for $m_{c}=1.3(1.7) \mathrm{GeV}$. The uncertainties in the calculations indicate that it is not possible to determine the mass of the charm quark simply by measuring the total charm cross section.

\section{Conclusions}

The cross section for inclusive charm production in two-photon collisions, $\sigma\left(\mathrm{e}^{+} \mathrm{e}^{-} \rightarrow \mathrm{e}^{+} \mathrm{e}^{-} \mathrm{c} \bar{c} \mathrm{X}\right)$, is measured with the $\mathrm{L} 3$ detector at $91 \mathrm{GeV} \leq \sqrt{\mathrm{s}} \leq$ $183 \mathrm{GeV}$. The cross section increases with energy as expected by QCD predictions.

The direct process $\gamma \gamma \rightarrow \mathrm{c} \overline{\mathbf{c}}$ is insufficient to describe the data, even if real and virtual gluon corrections are included. The cross sections and the event distributions require contributions from the resolved processes which are dominantly $\gamma \mathrm{g} \rightarrow \mathrm{c} \overline{\mathbf{c}}$. The data therefore require a significant gluon content in the photon. 


\section{Acknowledgements}

We wish to express our gratitude to the CERN accelerator divisions for the excellent performance of the LEP machine. We also acknowledge and appreciate the effort of all the engineers, technicians and support staff who have participated in the construction and maintenance of this experiment. We would like to thank Alex Finch and Michael Krämer for providing us with the program for the cross section calculation.

\section{References}

[1] M. Drees, M. Krämer, J. Zunft, P.M. Zerwas, Phys. Lett. B 306 (1993) 371.

[2] JADE Collab., W. Bartel et al., Phys. Lett. B 184 (1987) 288.

[3] TPC/Two-Gamma Collab., M. Alston-Garnjost et al., Phys. Lett. B 252 (1990) 499.

[4] TASSO Collab., W. Braunschweig et al., Z. Phys. C 47 (1990) 499.

[5] TOPAZ Collab., R. Enomoto et al., Phys. Lett. B 328 (1994) 535; Phys. Rev. D 50 (1994) 1879; Phys. Lett. B 341 (1994) 99; B 341 (1994) 238.

[6] VENUS Collab., S. Uehara et al., Z. Phys. C 63 (1994) 213.

[7] AMY Collab., T. Aso et al., Phys. Lett. B 363 (1995) 249; B 381 (1996) 372.

[8] ALEPH Collab., D. Buskulic et al., Phys. Lett. B 355 (1995) 595.
[9] L3 Collab., B. Adeva et al., Nucl. Instr. Meth. A 289 (1990) 35; M. Acciarri et al., Nucl. Instr. Meth. A 351 (1994) 300; M. Chemarin et al., Nucl. Instr. Meth. A 349 (1994) 345; I.C. Brock et al., Nucl. Instr. Meth. A 381 (1996) 236; A Adam et al., Nucl. Instr. Meth. A 383 (1996) 342.

[10] J.A.M. Vermaseren, Nucl. Phys. B 229 (1983) 347.

[11] T. Sjöstrand, Comput. Phys. Commun. 39 (1986) 347; T. Sjöstrand, M. Bengtsson, Comput. Phys. Commun. 43 (1987) 367.

[12] T. Sjöstrand, Comput. Phys. Commun. 82 (1994) 74.

[13] S. Jadach, B.F.L. Ward, Z. Was, Comput. Phys. Commun. 79 (1994) 503

[14] M. Skrzypek, S. Jadach, W. Placzek, Z. Was, Comput. Phys. Commun. 94 (1996) 216.

[15] M. Cacciari, Nucl. Phys. B 466 (1996) 173.

[16] G.A. Schuler, T. Sjöstrand, Z. Phys. C 68 (1995) 607; Phys. Lett. B 376 (1996) 193.

[17] V.M. Budnev et al., Phys. Rep. 15 (1975) 181.

[18] The L3 detector simulation is based on GEANT Version 3.14; see R. Brun et al., GEANT 3, CERN DD/EE/84-1 (Revised), September 1987 and the GHEISHA program (H. Fesefeld, RWTH Aachen Report PITHA85/02 (1985)) for the simulation of hadronic interactions.

[19] The LEP Experiments: ALEPH, DELPHI, L3 and OPAL, Nucl. Instr. Meth. A 378 (1996) 101.

[20] V.P. Andreev, R.R. McNeil, A.L. Stone, L3 Note 2263 (1998). This L3 Note is available on request from: The L3 secretariat, CERN, CH-1211 Geneva 23, Switzerland. Internet: http://13www.cern.ch

[21] M. Cacciari et al., in: G. Altarelli, CERN 96-01 Physics at LEP2, 1996, vol. 1, p. 334.

[22] M. Glück, E. Reya, A. Vogt, Phys. Rev. D 46 (1992) 1973.

[23] M. Drees, K. Grassie, Z. Phys. C 28 (1985) 451. 